\title{
WELL-POSEDNESS OF A THERMO-MECHANICAL MODEL FOR SHAPE MEMORY ALLOYS UNDER TENSION
}

\author{
Pavel KrejČí ${ }^{1}$ and Ulisse Stefanelli ${ }^{2}$
}

\begin{abstract}
We present a model of the full thermo-mechanical evolution of a shape memory body undergoing a uniaxial tensile stress. The well-posedness of the related quasi-static thermo-inelastic problem is addressed by means of hysteresis operators techniques. As a by-product, details on a time-discretization of the problem are provided.
\end{abstract}

Mathematics Subject Classification. 74N30, 74C05, 35K55.

Received July 9, 2009. Revised December 14, 2009.

Published online March 17, 2010.

\section{INTRODUCTION}

Shape memory alloys (SMAs) belong to the general class of so-called smart materials: their ability to completely recover comparably large deformations has attracted an increasing attention in the last decades [15,16]. Within a suitable high-temperature range, SMAs are super-elastic, namely they fully recover mechanical deformations up to 5-8\% (ordinary steels plasticize around 1\%). At lower temperature regimes deformations are permanent but can still be recovered by means of a thermal treatment (heating). This is the so-called shape memory effect. Both these effect are nowadays exploited in a variety of different technological contexts ranging from Aerospace, to Earthquake, to Biomechanical Engineering. New applications of SMAs are constantly emerging. This fact triggers an intense research in the direction of the efficient description of the corresponding material behavior.

The Engineering and Materials literature on SMAs models is vast and it is completely beyond our purposes even to attempt a review. Indeed, SMA behavior has been investigated at all scales (microscopic, mesoscopic with volume fractions, macroscopic) and by means of a full menagerie of modelling perspectives. Even restricting to the realm of macroscopic-phenomenological models (which is the focus of this paper), the different modelling options available are many and diversified and the corresponding cross-validation is still under assessment $[6,18$, $19,21,22,27,28,35-39,42,43]$. On the contrary, the mathematical treatment of full thermo-mechanical problems for SMAs is less developed for the only comprehensive results in this sense refer to either the original formulations or modifications of the Frémond [19] and the Falk and Konopka [17,18] models. With no claim of completeness, the reader is referred to $[1,2,12-14,24,34,45]$ and the related references for a comprehensive collection of results.

\footnotetext{
Keywords and phrases. Shape memory alloys, thermo-mechanics, well-posedness, hysteresis operator.

${ }^{1}$ Matematický ústav AV ČR, Žitná 25, 11567 Praha 1, Czech Republic. krejci@math.cas.cz;

http://www.math.cas.cz/ ${ }^{\sim} k r e j c i /$

${ }^{2}$ IMATI - CNR, via Ferrata 1, 27100 Pavia, Italy. stefanelli@imati.cnr.it; http://www.imati.cnr.it/ulisse
} 
We shall instead focus here on the phenomenological model for polycrystalline materials originally advanced by Souza et al. [40] and subsequently refined by Auricchio and Petrini [3-5] (the Souza-Auricchio model in the following). This model shows some distinctive advantage with respect to former contributions in terms both of simplicity (8 easily fitted material parameters are required for the full 3D thermo-mechanical description), robustness with respect to discretizations, and ability to capture experimental evidence. These desirable features are distinguishing the Souza-Auricchio model with respect to competitors and have recently attracted a growing attention in the SMA Engineering community. The aim of this note is to progress the mathematical understanding of the Souza-Auricchio model in the direction of the full thermo-mechanically coupled situation of thermal and stress-induced transformations.

The isothermal case of the Souza-Auricchio has been already addressed from the mathematical and numericaltheoretical viewpoints in [9] and [32,33], respectively, and some extension to even more detailed material behaviors has been advanced in $[7,8,10,11]$. As regards, the non-isothermal situation, one has to mention the papers by Mielke et al. [30,31] where the temperature of the specimen is assumed to be changing in time, being however given a-priori.

The first existence result of the full thermo-mechanical quasi-static evolution of a SMA body governed by the Souza-Auricchio model has been provided by these authors in [26]. A crucial point of [26] is however the remark that the original Souza-Auricchio modelling choice is not completely satisfactory from the thermodynamical viewpoint. The main drawback of this fact is that the corresponding quasi-static thermo-inelastic evolution PDE problem is generally ill-posed. The focus in [26] is hence on a slight modification of the original SouzaAuricchio model which under reasonable restrictions on material parameters entails existence of strong solutions by means of a space-approximation procedure. However, no uniqueness proof nor time-discretization procedure are provided in [26].

This note continues the discussion on the fully thermo-mechanically coupled situation by advancing an alternative modification of the original Souza-Auricchio model. We reduce a priori our model to the uniaxial tensile stress situation, namely we assume $\sigma \geq 0$ ( $\sigma$ is the uniaxial tension) and we present a new thermodynamically consistent model in Section 2. The corresponding PDE system given by the energy and momentum balance (here in a quasi-stationary form) and the material constitutive relation is proved to admit a unique solution under no restrictions on material parameters (Sect. 3). In particular, with respect to the model in [26], the present framework appears to be more robust with respect to parameter changes. An interesting by-product of our existence theory consists in the proof of the convergence of a suitable time-discretization procedure. This in particular paves the way to some possible future numerical validation of the model. Finally, differently from [26], we are here able to provide a continuous-dependence result.

Let us mention that the uniaxial tensile stress situation here considered is by far the reference test-case with respect to experiments on SMA. In particular, our reduction assumption to tension tests completely covers the situation of SMA wires (which are clearly not tested under compression). The extension of our results to the compression case is not immediate and deserves some further consideration which we plan to develop elsewhere.

\section{THERMO-MECHANICAL MODEL}

\subsection{SMA behavior}

The amazing thermo-mechanical behavior of SMAs is commonly interpreted as the effect of an abrupt structural phase transition at the metallic lattice level between a highly symmetric crystallographic phase called austenite (mostly cubic, predominant at higher temperatures) and less symmetric phases called martensites (different variants due to symmetry breaking, energetically favorable at lower temperatures).

By cooling down a fully austenitic specimen below some critical temperature, the material undergoes a solidsolid second order phase transformation toward a finely structured martensitic phase. This is the so-called multi-variant martensite (also called twinned or non-oriented) which roughly corresponds to a balanced local mixture of many martensitic variants. By keeping the temperature constant (and suitably low) and applying an external stress, one specific martensitic variant turns out to be more favorable and the whole multi-variant 
martensite progressively transforms into the so-called single-variant martensite (detwinned, oriented). This fact gives rise to a macroscopic deformation due to the specific asymmetry of the selected variant of martensite. The latter phase transformation is generically assumed to be of the first order (no latent heat) and irreversible: at sufficiently low temperatures, the single-variant martensite does not transform back to the multi-variant martensite upon unloading.

\subsection{Thermodynamic potentials}

We assume that the SMA specimen has a constant mass density (normalized to 1) and that the reference configuration can be assimilated to the interval $\Omega=[0, \ell]$. The evolution will be completely described by means of three state variables: the absolute temperature $\theta>0$, the tensile stress $\sigma \geq 0$, and the local proportion of single-variant martensite $\chi \in[0,1]$.

We prescribe the Gibbs energy density function in the form [20,29]

$$
G(\theta, \sigma, \chi) \doteq-c_{0} \theta(\log \theta-1)-\frac{\sigma^{2}}{2 E}-\varepsilon_{*} \sigma \chi+\frac{L}{\theta_{*}}\left(\theta-\theta_{*}\right) \chi+\frac{E_{h}}{2} \chi^{2}+I_{[0,1]}(\chi)
$$

where $c_{0}>0$ denotes the specific heat density, $E>0$ stands for the elasticity modulus, $E_{h}>0$ is a hardening modulus for the proportion of oriented martensite $\chi$, and $\varepsilon_{*}>0$ represents the maximal strain which is obtainable by martensitic reorientation in the material. The function $I_{[0,1]}$ is the indicator function of the interval [0,1], namely $I_{[0,1]}(r) \doteq 0$ if $r \in[0,1]$ and $I_{[0,1]}(r) \doteq \infty$ elsewhere. The constant $L>0$ is the austenite-martensite latent heat density, and $\theta_{*}>0$ is a reference temperature for the austenite-martensite phase transition at zero stress.

The corresponding specific entropy $s$, strain $\varepsilon$, and specific internal energy $U$ are given by

$$
\begin{aligned}
s & \doteq-\frac{\partial G}{\partial \theta}=c_{0} \log \theta-\frac{L}{\theta_{*}} \chi \\
\varepsilon & \doteq-\frac{\partial G}{\partial \sigma}=\frac{\sigma}{E}+\varepsilon_{*} \chi \\
U & \doteq \theta s+\sigma \varepsilon+G=c_{0} \theta+\frac{\sigma^{2}}{2 E}+\frac{E_{h}}{2} \chi^{2}-L \chi+I_{[0,1]}(\chi)
\end{aligned}
$$

\subsection{Flow rule}

The evolution of the material is assumed to show a dissipative character in the dynamic of the internal variable $\chi$. In particular, the dissipation is assumed to be rate-independent and in the form

$$
\varphi\left(\chi_{t}\right) \doteq\left(\sigma_{*}+L\right)\left(\chi_{t}\right)^{+}
$$

with a constant $\sigma_{*}>0$ representing the switching stress between different martensite orientations. The evolution of $\chi$ is hence driven by the flow rule

$$
0 \in \partial_{\chi_{t}} \varphi\left(\chi_{t}\right)+\partial_{\chi} G(\theta, \sigma, \chi)
$$

where the symbols $\partial_{\chi_{t}}$ and $\partial_{\chi}$ stand for the subdifferential in the sense of Convex Analysis with respect to the indicated variables. By explicitly computing

$$
\partial_{\chi} G=-\varepsilon_{*} \sigma+\frac{L}{\theta_{*}}\left(\theta-\theta_{*}\right)+E_{h} \chi+\partial I_{[0,1]}(\chi)
$$




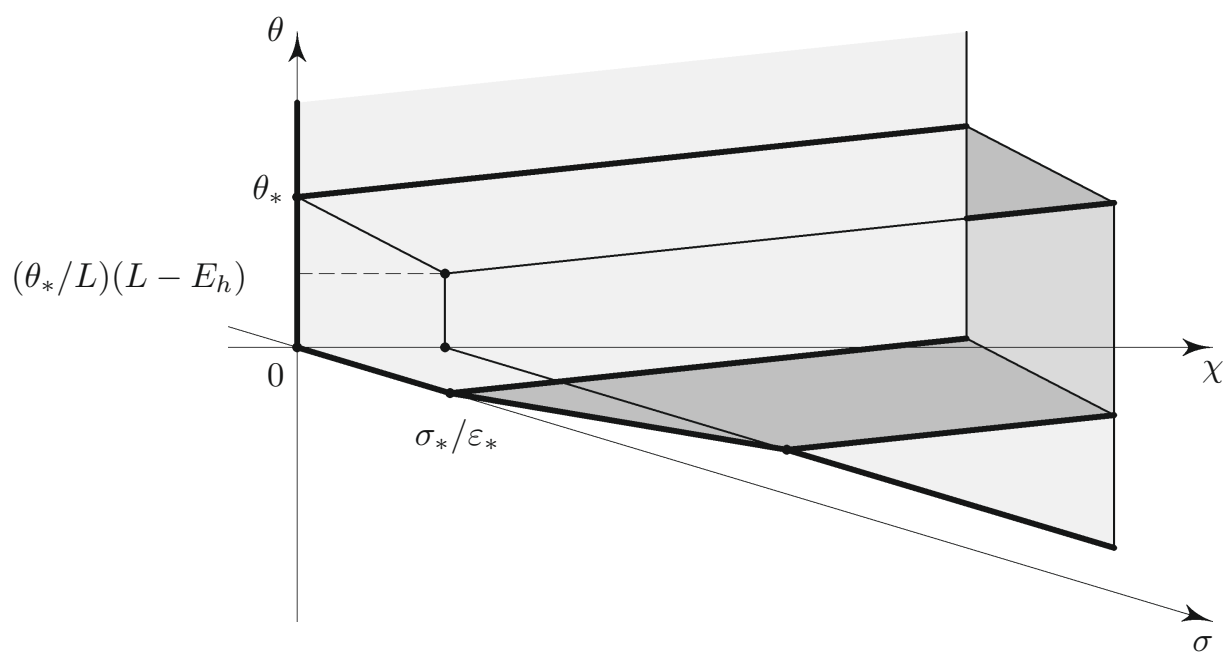

Figure 1. Admissible state set.

inclusion (2.4) can be equivalently rewritten in the form

$$
\varepsilon_{*} \sigma-\frac{L}{\theta_{*}}\left(\theta-\theta_{*}\right) \in\left(\sigma_{*}+L\right) H\left(\chi_{t}\right)+E_{h} \chi+\partial I_{[0,1]}(\chi) .
$$

Here, $H$ is the maximal monotone Heaviside graph, namely $H(r) \doteq r /|r|$ for $r \neq 0$ and $H(0) \doteq[0,1]$.

\subsection{Thermodynamic consistency}

The above introduced model turns out to be consistent with the Second Law of Thermodynamics for the pointwise validity of the Clausius-Duhem inequality

$$
-G_{t}-\theta_{t} s-\sigma_{t} \varepsilon-q \frac{\theta_{x}}{\theta} \geq 0
$$

can be checked (at least formally). Let us choose from the very beginning that the heat flux $q$ obeys Fourier's law $q \doteq-\kappa \theta_{x}(\kappa>0)$ and note that the solution temperature $\theta$ turns out to be positive for all times (see (3.13) below). Then, by using the constitutive relations (2.1)-(2.2), one directly computes that

$$
\begin{aligned}
-G_{t}-\theta_{t} s-\sigma_{t} \varepsilon-q \frac{\theta_{x}}{\theta} & =-\frac{\partial G}{\partial \theta} \theta_{t}-\frac{\partial G}{\partial \sigma} \sigma_{t}-\frac{\partial G}{\partial \chi} \chi_{t}-\theta_{t} s-\sigma_{t} \varepsilon-q \frac{\theta_{x}}{\theta} \\
& =-\frac{\partial G}{\partial \chi} \chi_{t}+\kappa \frac{\theta_{x}^{2}}{\theta} \stackrel{(2.4)}{=} \partial \varphi\left(\chi_{t}\right) \chi_{t}+\kappa \frac{\theta_{x}^{2}}{\theta}=\varphi\left(\chi_{t}\right)+\kappa \frac{\theta_{x}^{2}}{\theta} \stackrel{\theta>0}{\geq} 0 .
\end{aligned}
$$

\subsection{Admissible states}

The material constitutive relation (2.5) entails some restriction on the admissible values for the state quantities $\theta, \sigma$, and $\chi$. In particular, the set of admissible values is represented in Figure 1.

Owing to the presence of the constraint $I_{[0,1]}(\chi)$, we always have $\chi \in[0,1]$. For large temperatures, i.e. $\theta>\theta_{*}\left(1+\left(\varepsilon_{*} \sigma\right) / L\right)$, the only admissible value for $\chi$ is 0 and all the material is in the austenitic phase. On the contrary, for $\theta<\left(\theta_{*} / L\right)\left(\varepsilon_{*} \sigma-\sigma_{*}-E_{h}\right)^{+}$, the material is all in the oriented martensitic phase. In the interior of the above-depicted tunnel-like region we have that $\chi$ is constant.

We shall assume from the very beginning that

$$
E_{h}<L
$$




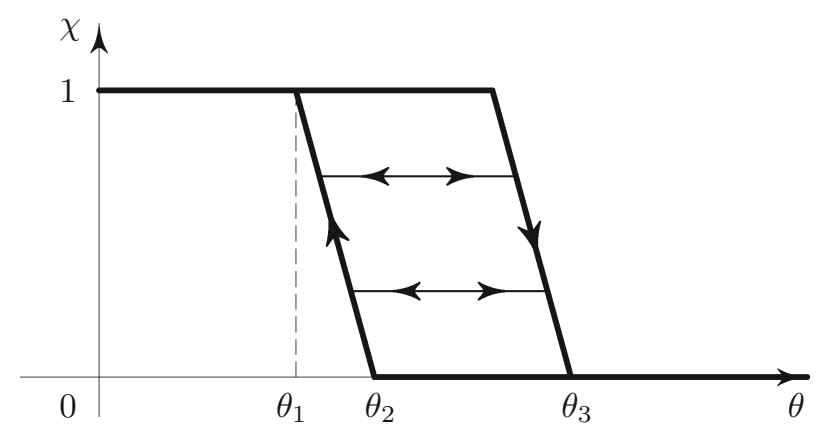

FIGURE 2. The temperature-phase dependence at a fixed high stress.

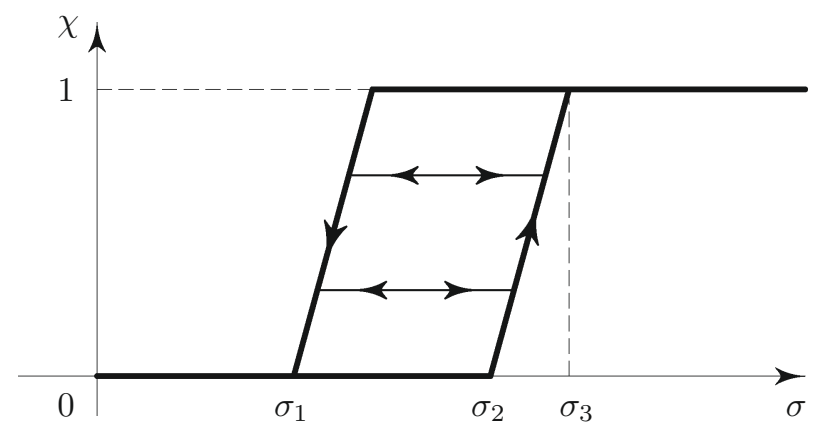

FiguRE 3. The stress-phase dependence at a fixed high temperature.

Indeed, a direct inspection of the diagram in Figure 1 reveals that if this was not the case, a completely oriented crystal at zero stress would not be admissible but, possibly, at a temperature of $0 \mathrm{~K}$. This clearly contradicts experience as single martensitic crystals may be observed at room temperature. Hence, let us stress that (2.7) is not a restriction on the possible choice of material parameters (see the Introduction) but rather a binding requirement for the model in order to make sense.

Figures 2 and 3 show the constitutive behavior at constant high stress and constant high temperature. The critical stress and temperature values in Figures 2 and 3 are

$$
\begin{aligned}
& \theta_{1}=\frac{\theta_{*}}{L}\left(\varepsilon_{*} \sigma-\sigma_{*}-E_{h}\right), \\
& \theta_{2}=\frac{\theta_{*}}{L}\left(\varepsilon_{*} \sigma-\sigma_{*}\right), \\
& \theta_{3}=\frac{\theta_{*}}{L}\left(\varepsilon_{*} \sigma+L\right), \\
& \sigma_{1}=\frac{1}{\varepsilon_{*}}\left(\frac{L \theta}{\theta_{*}}-L\right), \\
& \sigma_{2}=\frac{1}{\varepsilon_{*}}\left(\frac{L \theta}{\theta_{*}}+\sigma_{*}\right), \\
& \sigma_{3}=\frac{1}{\varepsilon_{*}}\left(\frac{L \theta}{\theta_{*}}+\sigma_{*}+E_{h}\right) .
\end{aligned}
$$

\subsection{Reformulation of the constitutive relation}

The crucial step in our analysis is the equivalent reformulation of the constitutive relation (2.5) in the form of a hysteresis operator.

Recall that the play operator $\mathfrak{p}_{[a, b]}:[a, b] \times W^{1,1}(0, T) \rightarrow W^{1,1}(0, T):\left(z_{0}, v\right) \mapsto \xi=\mathfrak{p}_{[a, b]}\left[z_{0}, v\right]$ associated with a fixed interval $[a, b]$ (the so-called characteristic interval) is defined by solving the variational inequality

$$
\left\{\begin{array}{l}
v(t)-\xi(t) \in[a, b] \quad \forall t \in[0, T] \\
v(0)-\xi(0)=z_{0}, \\
\dot{\xi}(t)(v(t)-\xi(t)-y) \geq 0 \quad \text { for a.e. } t, \forall y \in[a, b] .
\end{array}\right.
$$


The reader is referred to $[12,25,44]$ for a reference on the mathematical theory of hysteresis and, in particular, for a full discussion on the properties of the play operator.

By means of the above-mentioned operator, we briefly show that inclusion (2.5) is equivalent to

$$
\chi=Q\left(\frac{1}{E_{h}} \mathfrak{p}_{\left[0, \sigma_{*}+L\right]}\left[\varepsilon_{*} \sigma-\frac{L}{\theta_{*}}\left(\theta-\theta_{*}\right)\right]\right)
$$

where $Q(z)=\max \{0, \min \{z, 1\}\}$ is the projection of $\mathbb{R}$ onto $[0,1]$ and $\mathfrak{p}_{\left[0, \sigma_{*}+L\right]}$ is the play operator with characteristic interval $\left[0, \sigma_{*}+L\right]$. Set $v \doteq \varepsilon_{*} \sigma-\left(L / \theta_{*}\right)\left(\theta-\theta_{*}\right)$. Note that, for the sake of notational simplicity, in relation (2.9) we have omitted the indication of the initial value

$$
z_{0}=v(0)-\chi(0)=\varepsilon_{*} \sigma(0)-\left(L / \theta_{*}\right)\left(\theta(0)-\theta_{*}\right)-\chi(0)
$$

For any selection $\xi \in E_{h} \chi+\partial I_{[0,1]}(\chi)$, we rewrite $(2.5)$ equivalently as

$$
\chi=Q\left(\frac{1}{E_{h}} \xi\right), \quad v-\xi \in\left[0, \sigma_{*}+L\right], \quad \chi_{t}(v-\xi-y) \geq 0 \text { a.e. } \forall y \in\left[0, \sigma_{*}+L\right]
$$

Formula (2.9) thus gives one particular solution of (2.5). On the other hand, relation (2.10) determines $\chi$ uniquely: if $\left(\chi_{1}, \xi_{1}\right),\left(\chi_{2}, \xi_{2}\right)$ both satisfy $(2.10)$ for the same input $v$, then $\left(\chi_{1}-\chi_{2}\right)_{t}\left(\xi_{1}-\xi_{2}\right) \leq 0$ a.e., hence $\left(\chi_{1}-\chi_{2}\right)_{t}\left(\chi_{1}-\chi_{2}\right) \leq 0$ a.e., and we conclude that $\chi_{1}=\chi_{2}$. This proves the equivalence of $(2.9)$ and $(2.5)$.

\subsection{Balance equations}

The energy balance of the specimen reads

$$
U_{t}+q_{x}=\sigma \varepsilon_{t}+r(\theta, x, t)
$$

The function $r(\theta, x, t)$ stands for some heat source and the explicit $\theta$-dependence is intended to model the case under which the temperature distribution influences the external thermal actions along the body.

Owing to the above positions, we can rewrite the energy balance in terms of the state variables in the form

$$
c_{0} \theta_{t}-\kappa \theta_{x x}=\left(L \chi-\frac{E_{h}}{2} \chi^{2}\right)_{t}+\varepsilon_{*} \sigma \chi_{t}+r(\theta, x, t) .
$$

Note that no time derivative of the constraint $\partial_{t} I_{[0,1]}(\chi)$ appears in the latter. Indeed, all admissible trajectories necessarily have finite internal energy. In particular, $\chi(t) \in[0,1]$ for all times. Consequently, $I_{[0,1]}(\chi(t))=0$ everywhere and $\partial_{t} I_{[0,1]}(\chi)=0$.

Equation (2.7) has to be coupled with suitable boundary conditions. We shall ask here for no-flux conditions on $\theta$ (homogeneous Neumann)

$$
\theta_{x}(0, t)=\theta_{x}(\ell, t)=0,
$$

but note that other choices may be considered as well.

As for the momentum balance of the body we assume quasi-stationarity and reduce to

$$
\sigma_{x}=0, \quad u(0, t)=0, \quad \sigma(\ell)=g(t)
$$


for the displacement $u$ and some given traction $g$. In particular, we can immediately solve for $\sigma(t)$ (space homogeneous) and consider it to be a datum in the following. Note that there would be no particular intricacy in considering the situation $\sigma_{x}+b=0$ for some given body force $b$.

\subsection{Notation}

Henceforth we will use the symbol $c$ for any positive constant depending on data only. The value $c$ may possibly change from line to line, even within the same chain of inequalities. In places we shall explicitly specify dependencies of $c$ upon data.

\section{Well-POSEDNESS RESUlt}

We shall use the symbols $(\cdot, \cdot)$ and $\|\cdot\|$ in order to indicate the usual scalar product and the corresponding norm in $L^{2}(\Omega)$. Moreover, $\|\cdot\|_{E}$ stands for the norm in the generic normed space $E$. Let us recall that all constants $c_{0}, E, \varepsilon_{*}, \theta_{*}, L, E_{h}$ are assumed to be positive and enlist our assumptions as follows.

$$
\begin{aligned}
& E_{h}<L, \\
& \sigma \in H^{1}(0, T), \quad \sigma \geq 0, \\
& \theta_{0} \in H^{1}(\Omega), \quad \theta_{0}>0, \quad \chi_{0} \in[0,1] \text { a.e., } \\
& r: \mathbb{R} \times \Omega \times[0, T] \rightarrow \mathbb{R} \quad \text { is a Carathéodory function with } \\
& r(0, \cdot, \cdot) \in L^{2}(\Omega \times(0, T)), \\
& \exists \Lambda_{r}>0:\left|r\left(\theta_{1}, \cdot, \cdot\right)-r\left(\theta_{2}, \cdot, \cdot\right)\right| \leq \Lambda_{r}\left|\theta_{1}-\theta_{2}\right| \text { a.e., } \forall \theta_{1}, \theta_{2}>0, \\
& \exists \theta_{r}>0: r(\theta, \cdot, \cdot) \geq 0 \text { a.e., } \forall \theta \leq \theta_{r} .
\end{aligned}
$$

Within the frame of Section 2.7, the regularity (3.2) of the tension $\sigma$ follows at once if $g \in H^{1}(0, T)$. Assumption (3.6) expresses the fact that external heat sources should be prevented from cooling an already very cold body. Here is our main result.

Theorem 3.1 (well-posedness). Assume (3.1)-(3.6). Then, there exists a unique pair $(\theta, \chi)$ such that

$$
\begin{aligned}
& \theta \in H^{1}\left(0, T ; L^{2}(\Omega)\right) \cap L^{2}\left(0, T ; H^{2}(\Omega)\right), \\
& \chi \in H^{1}\left(0, T ; L^{2}(\Omega)\right) \cap L^{\infty}(\Omega \times(0, T)), \\
& c_{0} \theta_{t}-\kappa \theta_{x x}=\left(L \chi-\frac{E_{h}}{2} \chi^{2}\right)_{t}+\varepsilon_{*} \sigma \chi_{t}+r(\theta, \cdot, \cdot) \quad \text { a.e. }, \\
& \left(\sigma_{*}+L\right) H\left(\chi_{t}\right)+E_{h} \chi+\partial I_{[0,1]}(\chi) \ni \varepsilon_{*} \sigma-\frac{L}{\theta_{*}}\left(\theta-\theta_{*}\right) \quad \text { a.e. }, \\
& \theta_{x}(0, t)=\theta_{x}(\ell, t)=0 \quad \text { for a.e. } t \in(0, T), \\
& \theta(x, 0)=\theta_{0}(x), \quad \chi(x, 0)=\chi_{0}(x) \quad \text { for a.e. } x \in \Omega .
\end{aligned}
$$

Moreover, we have that

$$
\exists \underline{\theta}>0: \theta(x, t) \geq \underline{\theta} \quad \text { for all }(x, t) \in \Omega \times[0, T] .
$$

Finally, given two sets of data $\left(\sigma_{i}, \theta_{0 i}, \chi_{0 i}, r_{i}\right), i=1,2$ fulfilling (3.2)-(3.6) with

$$
\left\|\sigma_{i}\right\|_{H^{1}(0, T)}+\left\|\theta_{0 i}\right\|_{H^{1}(\Omega)}+\left\|r_{i}(0, \cdot, \cdot)\right\|_{L^{2}(\Omega \times(0, T))}+\Lambda_{r_{i}} \leq R
$$


for $i=1,2$ and some given $R>0$, the corresponding solutions $\left(\theta_{i}, \chi_{i}\right)$ satisfy the local Lipschitz continuous dependence estimate

$$
\begin{aligned}
\left\|\theta_{1}-\theta_{2}\right\|_{C\left([0, T] ; L^{1}(\Omega)\right)}+\left\|\chi_{1}-\chi_{2}\right\|_{C\left([0, T] ; L^{1}(\Omega)\right)} \leq & c(R)\left(\left\|\theta_{01}-\theta_{02}\right\|_{L^{1}(\Omega)}+\left\|\chi_{01}-\chi_{02}\right\|_{L^{1}(\Omega)}\right. \\
& \left.+\left\|\sigma_{1}-\sigma_{2}\right\|_{L^{2}(0, T)}+\sup _{|\theta| \leq c_{1}(R)}\left\|r_{1}(\theta)-r_{2}(\theta)\right\|_{L^{1}(\Omega \times(0, T))}\right)
\end{aligned}
$$

where $c, c_{1}:(0, \infty) \rightarrow(0, \infty)$ are suitable non-decreasing functions.

\section{Proof of Theorem 3.1}

\subsection{Change of variables}

The first step toward the proof of Theorem 3.1 consists in a useful reformulation of the original system (3.9)(3.10) in terms of the new variable

$$
v \doteq \varepsilon_{*} \sigma-\frac{L}{\theta_{*}}\left(\theta-\theta_{*}\right)
$$

which appears to be the input of the play operator in (2.9). The full quasi-static thermo-inelastic evolution problem (3.9)-(3.12) can hence be conveniently rewritten in terms of the variables $(v, \chi)$ as

$$
\begin{aligned}
& \bar{c}_{0} v_{t}+f(\chi)_{t}-\bar{\kappa} v_{x x}=-\varepsilon_{*} \sigma \chi_{t}+\bar{r}(v, \cdot) \quad \text { a.e., } \\
& \chi=Q\left(\frac{1}{E_{h}} \mathfrak{p}_{\left[0, \sigma_{*}+L\right]}[v]\right) \doteq \mathcal{F}\left[\chi_{0}, v\right] \quad \text { a.e., } \\
& v_{x}(0, t)=v_{x}(\ell, t)=0 \quad \text { for a.e. } t \in(0, T), \\
& v(x, 0)=v_{0}(x) \doteq \varepsilon_{*} \sigma(0)-\frac{L}{\theta_{*}}\left(\theta_{0}(x)-\theta_{*}\right), \quad \chi(x, 0)=\chi_{0}(x) \quad \text { for a.e. } x \in \Omega,
\end{aligned}
$$

where we have set, for notational simplicity, $\bar{c}_{0} \doteq c_{0} \theta_{*} / L, \bar{\kappa} \doteq \kappa \theta_{*} / L$ and

$$
f(\chi) \doteq L \chi-\frac{E_{h}}{2} \chi^{2}, \quad \bar{r}(v, x, t) \doteq-r\left(\frac{\theta_{*}}{L}\left(\varepsilon_{*} \sigma+L-v\right), x, t\right)+\frac{c_{0} \theta_{*}}{L} \varepsilon_{*} \sigma_{t}(t)
$$

Let us explicitly remark that $f$ is strongly monotone due to the fact that $E_{h}<L$ (see $\left.(2.7)\right)$ and $\chi \in[0,1]$.

We shall focus on proving well-posedness for the transformed problem (4.2)-(4.5) instead of the original system (3.9)-(3.12). This new problem turns out to be almost a quasilinear parabolic equation with hysteresis in the spirit of [44], Problem 1.1, p. 260. Still, we cannot directly reduce to the known theory as we have to face the additional issues of an explicit time-dependence in the quasilinear hysteretic term as well as an extra semilinear term $\bar{r}(v)$. Hence, even by moving essentially in the frame of [44], Section IX.1, p. 258, we are forced to work out the few differences in the following.

Before moving on, we shall recall here some basic properties of $\mathcal{F}$ which are used below. The reader is referred to the cited monographs for proofs and extensions. 
Lemma 4.1 (properties of $\mathcal{F})$. Let the operator $\mathcal{F}: L^{2}\left(\Omega ; W^{1,1}(0, T)\right) \rightarrow L^{2}\left(\Omega ; W^{1,1}(0, T)\right)$ be defined as in (4.3). Then, given $\chi=\mathcal{F}\left[\chi_{0}, v\right]$, we have that

$$
\begin{aligned}
& \chi(t)=\mathcal{F}[\chi(s), v(\cdot+s)](t-s) \quad \forall 0 \leq s \leq t \leq T, \\
& \chi_{t} v_{t} \geq 0 \quad \text { a.e., } \\
& \mathcal{F} \text { is strongly continuous in } L^{2}(\Omega ; C([0, T])), \\
& \mathcal{F} \text { is bounded in } H^{1}\left(0, T ; L^{2}(\Omega)\right) .
\end{aligned}
$$

\subsection{Time-discretization}

Assume now to be given a partition of $[0, T]$ which we identify with the corresponding vector $\boldsymbol{\tau}=\left(\tau^{1}, \ldots, \tau^{N_{\tau}}\right)$ of strictly positive time steps. Note that we indicate with superscripts the elements of a generic vector. In particular $\tau^{j}$ represents the $j$-th component of the vector $\boldsymbol{\tau}$ (and not the $j$-th power of the scalar $\tau$ ).

We let $t_{\boldsymbol{\tau}}^{0}=0$ and recursively define

$$
t_{\boldsymbol{\tau}}^{i} \doteq t_{\boldsymbol{\tau}}^{i-1}+\tau^{i}, \quad I_{\boldsymbol{\tau}}^{i} \doteq\left(t_{\boldsymbol{\tau}}^{i-1}, t_{\boldsymbol{\tau}}^{i}\right] \quad \text { for } i=1, \ldots, N_{\boldsymbol{\tau}},
$$

and we will use the symbol $\bar{\tau} \doteq \max _{i=1, \ldots, N_{\tau}} \tau^{i}$ for the maximum time step (fineness of the partition). Moreover, we will make use of the notation $\left(u_{\tau}^{0}, \ldots, u_{\tau}^{N_{\tau}}\right)$ for generic vectors in $E^{N_{\tau}+1}$ ( $E$ being a normed space) and let $u_{\boldsymbol{\tau}}, \bar{u}_{\boldsymbol{\tau}}:[0, T] \rightarrow E$ be the corresponding piecewise affine and piecewise constant interpolants on the intervals $I_{\boldsymbol{\tau}}^{i}$, namely

$$
\begin{aligned}
& u_{\boldsymbol{\tau}}(0)=\bar{u}_{\boldsymbol{\tau}}(0) \doteq u_{\boldsymbol{\tau}}^{0}, \\
& u_{\boldsymbol{\tau}}(t) \doteq \alpha_{\boldsymbol{\tau}}^{i}(t) u_{\boldsymbol{\tau}}^{i}+\left(1-\alpha_{\boldsymbol{\tau}}^{i}(t)\right) u_{\boldsymbol{\tau}}^{i-1}, \quad \bar{u}_{\boldsymbol{\tau}}(t) \doteq u_{\boldsymbol{\tau}}^{i}
\end{aligned}
$$

where $\alpha_{\tau}^{i}(t) \doteq\left(t-t_{\tau}^{i-1}\right) / \tau^{i}$, for all $t \in I_{\tau}^{i}, i=1, \ldots, N_{\tau}$. Moreover, given $\left(u_{\tau}^{0}, \ldots, u_{\tau}^{N_{\tau}}\right)$ we define its timederivative $\left(\delta u_{\boldsymbol{\tau}}^{1}, \ldots, \delta u_{\boldsymbol{\tau} \tau}^{N_{\tau}}\right)$ as $\delta u_{\boldsymbol{\tau}}^{i} \doteq\left(u_{\boldsymbol{\tau}}^{i}-u_{\boldsymbol{\tau}}^{i-1}\right) / \tau^{i}, i=1, \ldots, N_{\boldsymbol{\tau}}$.

Our discretization of the system (4.2)-(4.5) reads as follows

$$
\begin{aligned}
& \bar{c}_{0} \delta v_{\boldsymbol{\tau}}^{i}+\delta(f(\chi))_{\boldsymbol{\tau}}^{i}-\bar{\kappa} v_{\boldsymbol{\tau}, x x}^{i}=-\varepsilon_{*} \sigma_{\boldsymbol{\tau}}^{i} \delta \chi_{\boldsymbol{\tau}}^{i}+\bar{r}_{\boldsymbol{\tau}}^{i}\left(v_{\boldsymbol{\tau}}^{i}\right) \quad \text { a.e. in } \Omega, i=1, \ldots, N_{\boldsymbol{\tau}}, \\
& \chi_{\boldsymbol{\tau}}^{i}=\mathcal{F}\left[\chi_{0}, v_{\boldsymbol{\tau}}\right]\left(t_{\boldsymbol{\tau}}^{i}\right) \quad \text { a.e. in } \Omega, i=1, \ldots, N_{\boldsymbol{\tau}}, \\
& v_{\boldsymbol{\tau}, x}^{i}(0)=v_{\boldsymbol{\tau}, x}^{i}(\ell)=0, \quad i=1, \ldots, N_{\boldsymbol{\tau}}, \\
& v_{\boldsymbol{\tau}}^{0}=v_{0}, \quad \chi_{\boldsymbol{\tau}}^{0}=\chi_{0} \quad \text { a.e. in } \Omega .
\end{aligned}
$$

Here $\sigma_{\boldsymbol{\tau}}^{i} \doteq \sigma\left(t_{\boldsymbol{\tau}}^{i}\right), \delta(f(\chi))_{\boldsymbol{\tau}}^{i}=\left(f\left(\chi_{\boldsymbol{\tau}}^{i}\right)-f\left(\chi_{\boldsymbol{\tau}}^{i-1}\right)\right) / \tau^{i}$ and, for all $(v, x) \in \mathbb{R} \times \Omega$ and $i=1, \ldots, N_{\boldsymbol{\tau}}$,

$$
\bar{r}_{\tau}^{i}(v, x) \doteq \frac{1}{\tau^{i}} \int_{t_{\tau}^{i-1}}^{t_{\tau}^{i}} \bar{r}(v, x, t) \mathrm{d} t .
$$

The existence and uniqueness of a solution $\left(\left(v_{\tau}^{0}, \chi_{\tau}^{0}\right), \ldots,\left(v_{\tau}^{N_{\tau}}, \chi_{\boldsymbol{\tau}}^{N_{\tau}}\right)\right) \in\left(H^{2}(\Omega) \times L^{2}(\Omega)\right)^{N_{\tau}+1}$ of the latter discrete problem can be recovered as in [44], p. 262. Indeed, by exploiting the so-called semigroup property (4.6), relation (4.11) entails that

$$
\chi_{\boldsymbol{\tau}}^{i}=\mathcal{F}\left[\chi_{\boldsymbol{\tau}}^{i-1}, v_{\boldsymbol{\tau}}\left(\cdot+t_{\boldsymbol{\tau}}^{i-1}\right)\right]\left(\tau^{i}\right) \doteq F_{\boldsymbol{\tau}}^{i}\left(v_{\boldsymbol{\tau}}^{i}\right)
$$

where $F_{\boldsymbol{\tau}}^{i}$ is bounded and continuous (see (4.8)-(4.9)). Hence, by collecting in the term $\rho_{\boldsymbol{\tau}}^{i}$ all the quantities which are know at time $t_{\tau}^{i-1}$, we can rewrite equation (4.10) as

$$
\bar{c}_{0} v_{\boldsymbol{\tau}}^{i}-\tau^{i} \bar{\kappa} v_{\boldsymbol{\tau}, x x}^{i}+f\left(F_{\boldsymbol{\tau}}^{i}\left(v_{\boldsymbol{\tau}}^{i}\right)\right)+\varepsilon_{*} \sigma_{\boldsymbol{\tau}}^{i} F_{\boldsymbol{\tau}}^{i}\left(v_{\boldsymbol{\tau}}^{i}\right)-\tau^{i} \bar{r}_{\boldsymbol{\tau}}^{i}\left(v_{\boldsymbol{\tau}}^{i}\right)=\rho_{\boldsymbol{\tau}}^{i} .
$$


Note that, by recalling the Lipschitz continuity of $r(3.5)$, we have that, for all $v_{1}, v_{2} \in \mathbb{R}$,

$$
\begin{aligned}
\left|\bar{r}_{\boldsymbol{\tau}}^{i}\left(v_{1}\right)-\bar{r}_{\boldsymbol{\tau}}^{i}\left(v_{2}\right)\right| \leq \frac{1}{\tau^{i}} \int_{t_{\boldsymbol{\tau}}^{i-1}}^{t_{\boldsymbol{\tau}}^{i}} \mid \bar{r}\left(v_{1}\right) & -\bar{r}\left(v_{2}\right) \mid \\
& \stackrel{(4.1)}{=} \frac{1}{\tau^{i}} \int_{t_{\tau}^{i-1}}^{t_{\tau}^{i}}\left|r\left(\frac{\theta_{*}}{L}\left(\varepsilon_{*} \sigma+L-v_{1}\right)\right)-r\left(\frac{\theta_{*}}{L}\left(\varepsilon_{*} \sigma+L-v_{2}\right)\right)\right| \stackrel{(3.5)}{\leq} \Lambda_{\bar{r}}\left|v_{1}-v_{2}\right|
\end{aligned}
$$

where we have set $\Lambda_{\bar{r}} \doteq \Lambda_{r} \theta_{*} / L$. Hence, relation (4.14) turns out to be an elliptic equation with a bounded and continuous (in $L^{2}(\Omega)$ ) perturbation $v \mapsto f\left(F_{\tau}^{i}(v)\right)+\varepsilon_{*} \sigma_{\tau}^{i} F_{\tau}^{i}(v)$ and a Lipschitz continuous (in $L^{2}(\Omega)$ ) perturbation $v \mapsto \tau^{i} \bar{r}_{\boldsymbol{\tau}}^{i}(v)$. Finally, by possibly taking $\overline{\boldsymbol{\tau}}$ small, equation (4.14) admits at least a solution.

\subsection{A priori estimates}

The next step consists in obtaining classical parabolic estimates independently of $\boldsymbol{\tau}$. Let us test equation (4.10) by $\delta v_{\tau}^{i}$ getting

$$
\bar{c}_{0}\left\|\delta v_{\boldsymbol{\tau}}^{i}\right\|^{2}+\left(\delta(f(\chi))_{\boldsymbol{\tau}}^{i}, \delta v_{\boldsymbol{\tau}}^{i}\right)+\bar{\kappa}\left(v_{\boldsymbol{\tau}, x}^{i}, \delta v_{\boldsymbol{\tau}, x}^{i}\right)=-\varepsilon_{*} \sigma_{\boldsymbol{\tau}}^{i}\left(\delta \chi_{\boldsymbol{\tau}}^{i}, \delta v_{\boldsymbol{\tau}}^{i}\right)+\left(\bar{r}_{\boldsymbol{\tau}}^{i}\left(v_{\boldsymbol{\tau}}^{i}\right), \delta v_{\boldsymbol{\tau}}^{i}\right) .
$$

The terms $\left(\delta(f(\chi))_{\boldsymbol{\tau}}^{i}, \delta v_{\boldsymbol{\tau}}^{i}\right)$ and $\varepsilon_{*} \sigma_{\boldsymbol{\tau}}^{i}\left(\delta \chi_{\boldsymbol{\tau}}^{i}, \delta v_{\boldsymbol{\tau}}^{i}\right)$ are non-negative due to the piecewise monotonicity property of (4.7) (see [44], (1.11)). Indeed, one has that

$$
\delta(f(\chi))_{\tau}^{i} \delta v_{\boldsymbol{\tau}}^{i} \geq 0 \quad \text { a.e. }
$$

as $f$ is non-decreasing (recall (3.1)) and we have that

$$
\delta(f(\chi))_{\boldsymbol{\tau}}^{i} \delta v_{\boldsymbol{\tau}}^{i}=\frac{\delta(f(\chi))_{\boldsymbol{\tau}}^{i}}{\delta \chi_{\boldsymbol{\tau}}^{i}} \delta \chi_{\boldsymbol{\tau}}^{i} \delta v_{\boldsymbol{\tau}}^{i} \stackrel{(4.7)}{\geq} 0 \quad \text { a.e. in }\left\{x \in \Omega \mid \delta \chi_{\boldsymbol{\tau}}^{i} \neq 0\right\}
$$

Then, by multiplying the above equation by $\tau^{i}$ and taking the sum for $i=1, \ldots, m \leq N_{\tau}$, we obtain

$$
\begin{aligned}
\sum_{i=1}^{m} \bar{c}_{0} \tau^{i}\left\|\delta v_{\boldsymbol{\tau}}^{i}\right\|^{2}+\frac{\bar{\kappa}}{2}\left\|v_{\boldsymbol{\tau}, x}^{m}\right\|^{2} \leq \frac{\bar{\kappa}}{2}\left\|v_{0, x}\right\|^{2}+\sum_{i=1}^{m} \tau^{i} \Lambda_{\bar{r}}\left\|v_{\boldsymbol{\tau}}^{i}\right\|\left\|\delta v_{\boldsymbol{\tau}}^{i}\right\|+\sum_{i=1}^{m} \tau^{i}\left(\bar{r}_{\boldsymbol{\tau}}^{i}(0), \delta v_{\boldsymbol{\tau}}^{i}\right) \\
\leq \frac{\bar{\kappa}}{2}\left\|v_{0, x}\right\|^{2}+\frac{\bar{c}_{0}}{2} \sum_{i=1}^{m} \tau^{i}\left\|\delta v_{\boldsymbol{\tau}}^{i}\right\|^{2}+\frac{1}{\bar{c}_{0}} \sum_{i=1}^{m} \tau^{i}\left\|\bar{r}_{\boldsymbol{\tau}}^{i}(0)\right\|^{2}+\frac{\Lambda_{\bar{r}}^{2}}{\bar{c}_{0}} \sum_{i=1}^{m} \tau^{i}\left\|v_{\boldsymbol{\tau}}^{i}\right\|^{2} \\
\leq \frac{\bar{\kappa}}{2}\left\|v_{0, x}\right\|^{2}+\frac{\bar{c}_{0}}{2} \sum_{i=1}^{m} \tau^{i}\left\|\delta v_{\boldsymbol{\tau}}^{i}\right\|^{2}+\frac{1}{\bar{c}_{0}} \sum_{i=1}^{m} \tau^{i}\left\|\bar{r}_{\boldsymbol{\tau}}^{i}(0)\right\|^{2}+\frac{\Lambda_{\bar{r}}^{2}}{\bar{c}_{0}} \sum_{i=1}^{m} \tau^{i}\left(2\left\|v_{0}\right\|^{2}+2 T \sum_{j=1}^{i} \tau^{j}\left\|\delta v_{\boldsymbol{\tau}}^{j}\right\|^{2}\right) .
\end{aligned}
$$

Now, by choosing $\overline{\boldsymbol{\tau}}$ sufficiently small in such a way that

$$
\frac{\Lambda_{\bar{r}}^{2}}{\bar{c}_{0}} \bar{\tau} 2 T<\frac{\bar{c}_{0}}{2},
$$

we can apply the discrete Gronwall lemma and obtain that

$$
\left\|v_{\boldsymbol{\tau}}\right\|_{H^{1}\left(0, T ; L^{2}(\Omega)\right) \cap L^{\infty}\left(0, T ; H^{1}(\Omega)\right)} \leq c
$$

where $c$ depends on $\left\|v_{0, x}\right\|$ and $\sum_{i=1}^{m} \tau^{i}\left\|\bar{r}_{\boldsymbol{\tau}}^{i}(0)\right\|^{2}$. Hence, by using the boundedness of $\mathcal{F}$ from (4.9) we also get that

$$
\left\|\chi_{\boldsymbol{\tau}}\right\|_{H^{1}\left(0, T ; L^{2}(\Omega)\right) \cap L^{\infty}(\Omega \times(0, T))} \leq c .
$$


Finally, by a comparison in equation (4.10) we have that

$$
-\bar{\kappa} \bar{v}_{\boldsymbol{\tau}, x x}=-\bar{c}_{0} v_{\boldsymbol{\tau}, t}-f(\chi)_{\boldsymbol{\tau}, t}-\varepsilon_{*} \bar{\sigma}_{\boldsymbol{\tau}} \chi_{\boldsymbol{\tau}, t}+\bar{r}_{\boldsymbol{\tau}}\left(\bar{v}_{\boldsymbol{\tau}}\right)
$$

where the subscript $t$ as in $v_{\boldsymbol{\tau}, t}$ stands for the time derivative and the right-hand side is bounded in $L^{2}(\Omega \times(0, T))$ independently of $\boldsymbol{\tau}$. Hence, by standard elliptic regularity we have that

$$
\left\|\bar{v}_{\boldsymbol{\tau}}\right\|_{L^{2}\left(0, T ; H^{2}(\Omega)\right)} \leq c .
$$

\subsection{Passage to the limit}

Estimates (4.15)-(4.17) entail that, upon extracting some (non relabeled) subsequences we have that, as $\tau \rightarrow 0$,

$$
\begin{aligned}
& v_{\boldsymbol{\tau}} \rightarrow v \quad \text { strongly in } L^{2}(\Omega ; C([0, T])) \text { and weakly in } H^{1}\left(0, T ; L^{2}(\Omega)\right) \cap L^{2}\left(0, T ; H^{2}(\Omega)\right), \\
& \bar{v}_{\boldsymbol{\tau}} \rightarrow v \quad \text { strongly in } L^{\infty}\left(0, T ; L^{2}(\Omega)\right) \text { and weakly in } L^{2}\left(0, T ; H^{2}(\Omega)\right), \\
& \chi_{\boldsymbol{\tau}} \rightarrow \chi \quad \text { weakly in } H^{1}\left(0, T ; L^{2}(\Omega)\right) .
\end{aligned}
$$

The strong convergence of $v_{\boldsymbol{\tau}}$ from (4.18) and the strong continuity of $\mathcal{F}$ in $L^{2}(\Omega ; C([0, T]))$ (see (4.8)) entail that, by letting $\xi_{\boldsymbol{\tau}}(t) \doteq \mathcal{F}\left[\chi_{0}, v_{\boldsymbol{\tau}}\right](t)$ (which is a priori not affine on the time-partition) we have that

$$
\xi_{\boldsymbol{\tau}} \rightarrow \mathcal{F}\left[\chi_{0}, v\right] \quad \text { strongly in } L^{2}(\Omega ; C([0, T])) .
$$

On the other hand, one has that $\xi_{\boldsymbol{\tau}}\left(t_{\boldsymbol{\tau}}^{i}\right) \equiv \chi_{\boldsymbol{\tau}}\left(t_{\boldsymbol{\tau}}^{i}\right)$ and $\xi_{\boldsymbol{\tau}}$ is bounded in $H^{1}\left(0, T ; L^{2}(\Omega)\right)$ independently of $\boldsymbol{\tau}$ by (4.9). It is hence a standard matter to conclude that

$$
\chi_{\boldsymbol{\tau}} \rightarrow \mathcal{F}\left[\chi_{0}, v\right] \quad \text { strongly in } L^{2}(\Omega ; C([0, T]))
$$

The boundary and initial conditions (4.4)-(4.5) directly pass to the limit. As for taking the limit in the equation

$$
\bar{c}_{0} v_{\boldsymbol{\tau}, t}+f(\chi)_{\boldsymbol{\tau}, t}-\bar{\kappa} \bar{v}_{\boldsymbol{\tau}, x x}=-\varepsilon_{*} \bar{\sigma}_{\boldsymbol{\tau}} \chi_{\boldsymbol{\tau}, t}+\bar{r}_{\boldsymbol{\tau}}\left(\bar{v}_{\boldsymbol{\tau}}\right)
$$

we just recall that $\bar{r}_{\boldsymbol{\tau}}\left(\bar{v}_{\boldsymbol{\tau}}\right) \rightarrow \bar{r}(v)$ strongly in $L^{2}(\Omega \times(0, T))$ [41], Lemma 7.1, and work out the term $f(\chi)_{\boldsymbol{\tau}, t}$ as

$$
f(\chi)_{\boldsymbol{\tau}, t}=f^{\prime}\left(\xi_{\boldsymbol{\tau}}\right) \chi_{\boldsymbol{\tau}, t}=f^{\prime}\left(\chi_{\boldsymbol{\tau}}\right) \chi_{\boldsymbol{\tau}, t}+\left(f^{\prime}\left(\xi_{\boldsymbol{\tau}}\right)-f^{\prime}\left(\chi_{\boldsymbol{\tau}}\right)\right) \chi_{\boldsymbol{\tau}, t}
$$

where $\xi_{\boldsymbol{\tau}}^{i}$ lies on the segment (in $L^{2}(\Omega)$ ) from $\chi_{\boldsymbol{\tau}}^{i-1}$ to $\chi_{\boldsymbol{\tau}}^{i}$. As $f^{\prime}$ is Lipschitz continuous with constant $E_{h}$, the convergence for $\chi_{\boldsymbol{\tau}}$ in $(4.21)$ entails that $f^{\prime}\left(\chi_{\boldsymbol{\tau}}\right) \chi_{\boldsymbol{\tau}, t} \rightarrow f^{\prime}(\chi) \chi_{t}$ weakly in $L^{2}(\Omega \times(0, T))$ (indeed, $f^{\prime}\left(\chi_{\boldsymbol{\tau}}\right)$ remain uniformly bounded in $L^{\infty}(\Omega \times(0, T)), f^{\prime}\left(\chi_{\boldsymbol{\tau}}\right) \rightarrow f^{\prime}(\chi)$ strongly, and $\chi_{\boldsymbol{\tau}, t} \rightarrow \chi_{t}$ weakly in $\left.L^{2}(\Omega \times(0, T))\right)$ whereas

$$
\left\|\left(f^{\prime}\left(\xi_{\boldsymbol{\tau}}\right)-f^{\prime}\left(\chi_{\boldsymbol{\tau}}\right)\right) \chi_{\boldsymbol{\tau}, t}\right\|_{L^{2}(\Omega \times(0, T))} \leq E_{h} \overline{\boldsymbol{\tau}}\left\|\chi_{\boldsymbol{\tau}, t}\right\|_{L^{2}(\Omega \times(0, T))} \rightarrow 0 .
$$

Finally, we have proved that $f(\chi)_{\boldsymbol{\tau}, t} \rightarrow f^{\prime}(\chi) \chi_{t}=f(\chi)_{t}$ weakly in $L^{2}(\Omega \times(0, T))$ and we are done.

\subsection{Uniqueness}

Let us start by recalling a crucial tool from [23].

Lemma 4.2 (Hilpert's inequality). Let $v_{1}, v_{2} \in W^{1,1}(0, T)$ and $z_{01}, z_{02} \in[a, b]$ be given, $\xi_{i}=\mathfrak{p}_{[a, b]}\left[z_{0 i}, v_{i}\right]$, $i=1,2$. Then for every nondecreasing Lipschitz continuous function $h: \mathbb{R} \rightarrow \mathbb{R}$ we have

$$
\frac{\mathrm{d}}{\mathrm{d} t}\left(h\left(\xi_{1}\right)-h\left(\xi_{2}\right)\right) \operatorname{sign}\left(v_{1}-v_{2}\right) \geq \frac{\mathrm{d}}{\mathrm{d} t}\left|h\left(\xi_{1}\right)-h\left(\xi_{2}\right)\right| \quad \text { a.e. }
$$


Proof. We have by the very definition (2.8) of play operator that

$$
\begin{aligned}
& \xi_{1, t}\left(\left(v_{1}-v_{2}\right)-\left(\xi_{1}-\xi_{2}\right)\right) \geq 0 \text { a.e., } \\
& \xi_{2, t}\left(\left(v_{2}-v_{1}\right)-\left(\xi_{2}-\xi_{1}\right)\right) \geq 0 \text { a.e. }
\end{aligned}
$$

Assume for instance that $\operatorname{sign}\left(v_{1}(t)-v_{2}(t)\right)>\operatorname{sign}\left(\xi_{1}(t)-\xi_{2}(t)\right)$. Then $v_{1}(t)-v_{2}(t)>\xi_{1}(t)-\xi_{2}(t)$ and $\xi_{1, t}(t) \geq 0, \xi_{2, t}(t) \leq 0$. Consequently,

$$
\begin{aligned}
& \frac{\mathrm{d}}{\mathrm{d} t} h\left(\xi_{1}\right)\left(\operatorname{sign}\left(v_{1}-v_{2}\right)-\operatorname{sign}\left(\xi_{1}-\xi_{2}\right)\right) \geq 0 \quad \text { a.e. } \\
& \frac{\mathrm{d}}{\mathrm{d} t} h\left(\xi_{2}\right)\left(\operatorname{sign}\left(v_{1}-v_{2}\right)-\operatorname{sign}\left(\xi_{1}-\xi_{2}\right)\right) \leq 0 \quad \text { a.e. }
\end{aligned}
$$

and the assertion follows.

Let us now assume to be given two strong solutions $\left(v_{1}, \chi_{1}\right)$ and $\left(v_{2}, \chi_{2}\right)$ of the system (4.2)-(4.5). Then, take the difference of the respective equations (4.2) and denote by $\tilde{v} \doteq v_{1}-v_{2}, \tilde{\chi} \doteq \chi_{1}-\chi_{2}, \tilde{f} \doteq f\left(\chi_{1}\right)-f\left(\chi_{2}\right)$, and $\tilde{r} \doteq \bar{r}\left(v_{1}\right)-\bar{r}\left(v_{2}\right)$, getting

$$
\bar{c}_{0} \tilde{v}_{t}+\tilde{f}_{t}-\bar{\kappa} \tilde{v}_{x x}=-\varepsilon_{*} \sigma \tilde{\chi}_{t}+\tilde{r} .
$$

By testing the above equation by $\operatorname{sign}(\tilde{v})$ and using $\left(\tilde{v}_{x x}, \operatorname{sign}(\tilde{v})\right) \leq 0$ and Lemma 4.2 along with the choice

$$
\xi \mapsto h(\xi) \doteq f\left(Q\left(\xi / E_{h}\right)\right) \equiv f(\chi)
$$

we have that

$$
\bar{c}_{0} \frac{\mathrm{d}}{\mathrm{d} t}\|\tilde{v}\|_{L^{1}(\Omega)}+\frac{\mathrm{d}}{\mathrm{d} t}\|\tilde{f}\|_{L^{1}(\Omega)} \leq-\varepsilon_{*} \sigma\left(\tilde{\chi}_{t}, \operatorname{sign}(\tilde{v})\right)+\Lambda_{\bar{r}}\|\tilde{v}\|_{L^{1}(\Omega)} \quad \text { a.e. in time. }
$$

The first term in the above right-hand side is to be handled again by Lemma 4.2 by choosing

$$
\xi \mapsto h(\xi) \doteq \varepsilon_{*} Q\left(\xi / E_{h}\right) \equiv \varepsilon_{*} \chi
$$

(note that for both choices (here and before (4.22)), the function $h$ is increasing and Lipschitz continuous) and using $\sigma \geq 0$ as

$$
-\varepsilon_{*} \sigma\left(\tilde{\chi}_{t}, \operatorname{sign}(\tilde{v})\right) \leq-\varepsilon_{*} \sigma \frac{\mathrm{d}}{\mathrm{d} t}\|\tilde{\chi}\|_{L^{1}(\Omega)} .
$$

Hence, by taking the integral of $(4.22)$ on $(0, t)$ we have that

$$
\bar{c}_{0}\|\tilde{v}(t)\|_{L^{1}(\Omega)}+\|\tilde{f}(t)\|_{L^{1}(\Omega)}+\int_{0}^{t} \varepsilon_{*} \sigma \frac{\mathrm{d}}{\mathrm{d} t}\|\tilde{\chi}\|_{L^{1}(\Omega)} \leq \Lambda_{\bar{r}} \int_{0}^{t}\|\tilde{v}\|_{L^{1}(\Omega)} .
$$

Now, from assumption (3.1) there exists $\rho>0$ such that

$$
\forall \chi \in[0,1]: \quad f^{\prime}(\chi) \geq \min _{x \in[0,1]} f^{\prime}(x)=\min _{\chi \in[0,1]}\left(L-E_{h} x\right)=L-E_{h} \geq \rho .
$$

Eventually, by integrating by parts we have that

$$
\bar{c}_{0}\|\tilde{v}(t)\|_{L^{1}(\Omega)}+\rho\|\tilde{\chi}(t)\|_{L^{1}(\Omega)}+\varepsilon_{*} \sigma(t)\|\tilde{\chi}(t)\|_{L^{1}(\Omega)} \leq \int_{0}^{t} \varepsilon_{*} \sigma_{t}\|\tilde{\chi}\|_{L^{1}(\Omega)}+\Lambda_{\bar{r}} \int_{0}^{t}\|\tilde{v}\|_{L^{1}(\Omega)}
$$

and $\tilde{v}=\tilde{\chi}=0$ follows by Gronwall. 


\subsection{Continuous dependence}

The uniqueness proof above can be further specified in order to provide a local Lipschitz continuous dependence estimate which is equivalent to (3.14). Let $\left(\sigma_{i}, r_{i}, \theta_{0 i}, \chi_{0 i}\right), i=1,2$, be two sets of data fulfilling assumptions (3.3)-(3.5). In particular, let

$$
\left\|\sigma_{i}\right\|_{H^{1}(0, T)}+\left\|\theta_{0 i}\right\|_{H^{1}(\Omega)}+\left\|r_{i}(0, \cdot, \cdot)\right\|_{L^{2}(\Omega \times(0, T))}+\Lambda_{r_{i}} \leq R
$$

for some $R>0$ and $i=1,2$. Correspondingly define $v_{0 i}$ and $\bar{r}_{i}$ as above and let $\left(v_{i}, \chi_{i}\right)$ be the solution of the system (4.2)-(4.5) for data $\left(\sigma_{i}, \bar{r}_{i}, v_{0 i}, \chi_{0 i}\right)$. Again, we shorten notations as $\tilde{v} \doteq v_{1}-v_{2}, \tilde{\chi} \doteq \chi_{1}-\chi_{2}, \tilde{\sigma} \doteq \sigma_{1}-\sigma_{2}$, $\tilde{f} \doteq f\left(\chi_{1}\right)-f\left(\chi_{2}\right)$, and $\tilde{r} \doteq \bar{r}_{1}\left(v_{1}\right)-\bar{r}_{2}\left(v_{2}\right)$. By taking the difference between relation (4.2) written for $i=1$ and the same relation for $i=2$, testing it on $\operatorname{sign}(\tilde{v})$, and exploiting Lemma 4.2 exactly as in Section 4.5 we get that

$$
\bar{c}_{0} \frac{\mathrm{d}}{\mathrm{d} t}\|\tilde{v}\|_{L^{1}(\Omega)}+\frac{\mathrm{d}}{\mathrm{d} t}\|\tilde{f}\|_{L^{1}(\Omega)}+\varepsilon_{*} \sigma_{1} \frac{\mathrm{d}}{\mathrm{d} t}\|\tilde{\chi}\|_{L^{1}(\Omega)} \leq-\varepsilon_{*} \tilde{\sigma}\left(\chi_{2, t}, \operatorname{sign}(\tilde{v})\right)+(\tilde{r}, \operatorname{sign}(\tilde{v})) \quad \text { a.e. }
$$

Upon integrating in time, the above left-hand side is to be handled as in Section 4.5. As for the right-hand side we argue as follows.

$$
\begin{aligned}
-\int_{0}^{t} \varepsilon_{*} \tilde{\sigma}\left(\chi_{2, t}, \operatorname{sign}(\tilde{v})\right)+\int_{0}^{t}(\tilde{r}, \operatorname{sign}(\tilde{v})) \leq \varepsilon_{*}\|\tilde{\sigma}\|_{L^{2}(0, T)}\left\|\chi_{2, t}\right\|_{L^{2}\left(0, T ; L^{1}(\Omega)\right)} & \\
& +\left\|\bar{r}_{1}\left(v_{1}\right)-\bar{r}_{2}\left(v_{1}\right)\right\|_{L^{1}(\Omega \times(0, T))}+\Lambda_{\bar{r}_{2}} \int_{0}^{t}\|\tilde{v}\|_{L^{1}(\Omega)} .
\end{aligned}
$$

Note that $\chi_{2, t}$ is bounded in $L^{2}\left(0, T ; L^{1}(\Omega)\right)$ in terms of data. Moreover, from estimate (4.15),

$$
\left\|v_{i}\right\|_{L^{\infty}(\Omega \times(0, T))} \leq c\left(v_{0 i}, \bar{r}_{i}(0, \cdot), \Lambda_{\bar{r}_{i}}\right) \doteq c_{1} .
$$

Hence, by Gronwall's lemma we get that

$$
\begin{aligned}
\|\tilde{v}\|_{C\left([0, T] ; L^{1}(\Omega)\right)}+\|\tilde{\chi}\|_{C\left([0, T] ; L^{1}(\Omega)\right)} \leq & c\left(v_{0 i}, \bar{r}_{i}(0, \cdot), \Lambda_{\bar{r}_{i}}, \sigma_{i}\right)\left(\|\tilde{v}(0)\|_{L^{1}(\Omega)}+\|\tilde{\chi}(0)\|_{L^{1}(\Omega)}\right. \\
& \left.+\|\tilde{\sigma}\|_{L^{2}(0, T)}+\sup _{|v| \leq c_{1}}\left\|r_{1}(v)-r_{2}(v)\right\|_{L^{1}(\Omega \times(0, T))}\right) .
\end{aligned}
$$

It now suffices to translate the latter back into the original variables $(\theta, \chi)$ in order to get the local Lipschitz continuity estimate (3.14).

\subsection{Positivity of the temperature}

Let us complete the proof of Theorem 3.1 by providing the strictly positive lower bound for the temperature in (3.13). To this end, by recalling (3.6), we set

$$
\underline{\theta} \doteq \min \left\{\min \theta_{0}, \theta_{r}, \frac{\theta_{*}}{L}\left(L-E_{h}\right)\right\}
$$

test the equation for $\theta(3.9)$ by $-(\theta-\underline{\theta})^{-} \doteq \min \{\theta-\underline{\theta}, 0\}$, and integrate in time getting

$$
\frac{\bar{c}_{0}}{2}\left\|(\theta-\underline{\theta})^{-}(t)\right\|^{2}+\kappa \int_{0}^{t}\left\|(\theta-\underline{\theta})_{x}^{-}\right\|^{2}=-\int_{0}^{t}\left(f^{\prime}(\chi) \chi_{t}+\varepsilon_{*} \sigma \chi_{t},(\theta-\underline{\theta})^{-}\right)-\int_{0}^{t}\left(r(\theta),(\theta-\underline{\theta})^{-}\right)
$$

where again $f(\chi)=L \chi-E_{h} \chi^{2} / 2$. 
We aim at showing that the above right-hand side is indeed non-positive. In fact, the term containing $r$ is non-positive due to assumption (3.6). Hence, as $f^{\prime}(\chi)+\varepsilon_{*} \sigma>0$ almost everywhere, we have that

$$
-\int_{0}^{t}\left(f^{\prime}(\chi) \chi_{t}+\varepsilon_{*} \sigma \chi_{t},(\theta-\underline{\theta})^{-}\right)-\int_{0}^{t}\left(r(\theta),(\theta-\underline{\theta})^{-}\right) \leq-\iint_{\left\{\chi_{t} \leq 0\right\}}\left(f^{\prime}(\chi) \chi_{t}+\varepsilon_{*} \sigma \chi_{t}\right)(\theta-\underline{\theta})^{-} .
$$

This last integrand differs from 0 only for $\theta<\underline{\theta} \leq \theta_{*}\left(L-E_{h}\right) / L$. On the other hand, by inspecting Figure 1 (or, more precisely, by analyzing the constitutive relation (2.5)) one realizes that

$$
\left(\theta<\frac{\theta_{*}}{L}\left(L-E_{h}\right), \quad \chi_{t} \leq 0\right) \quad \Longrightarrow \quad \chi \equiv 1
$$

As the set $\left\{\chi_{t}<0\right\} \cap\{\chi=1\}$ has clearly zero measure, we conclude that

$$
-\int_{0}^{t}\left(f^{\prime}(\chi) \chi_{t}+\varepsilon_{*} \sigma \chi_{t},(\theta-\underline{\theta})^{-}\right) \leq 0
$$

and the assertion follows from the fact that $(\theta-\underline{\theta})^{-}=0$.

Acknowledgements. This research has been performed during PK's visit in Pavia under the sponsorship of the ShortTerm Mobility program of the CNR. Partial supports from FP7-IDEAS-ERC-StG Grant \#200497 (BioSMA) and from the GAČR Grant P201/10/2315 are gratefully acknowledged.

\section{REFERENCES}

[1] T. Aiki, A model of 3D shape memory alloy materials. J. Math. Soc. Jpn. 57 (2005) 903-933.

[2] M. Arndt, M. Griebel and T. Roubíček, Modelling and numerical simulation of martensitic transformation in shape memory alloys. Contin. Mech. Thermodyn. 15 (2003) 463-485.

[3] F. Auricchio and L. Petrini, Improvements and algorithmical considerations on a recent three-dimensional model describing stress-induced solid phase transformations. Int. J. Numer. Methods Eng. 55 (2002) 1255-1284.

[4] F. Auricchio and L. Petrini, A three-dimensional model describing stress-temperature induced solid phase transformations. Part I: Solution algorithm and boundary value problems. Int. J. Numer. Methods Eng. 61 (2004) 807-836.

[5] F. Aurichio and L. Petrini, A three-dimensional model describing stress-temperature induced solid phase transformations. Part II: Thermomechanical coupling and hybrid composite applications. Int. J. Numer. Methods Eng. 61 (2004) 716-737.

[6] F. Auricchio and E. Sacco, A one-dimensional model for superelastic shape-memory alloys with different elastic properties between austenite and martensite. Int. J. Non-Linear Mech. 32 (1997) 1101-1114.

[7] F. Auricchio, A. Reali and U. Stefanelli, A phenomenological 3D model describing stress-induced solid phase transformations with permanent inelasticity, in Topics on Mathematics for Smart Systems (Rome, 2006), World Sci. Publishing (2007) 1-14.

[8] F. Auricchio, A. Reali and U. Stefanelli, A three-dimensional model describing stress-induced solid phase transformation with permanent inelasticity. Int. J. Plast. 23 (2007) 207-226.

[9] F. Auricchio, A. Mielke and U. Stefanelli, A rate-independent model for the isothermal quasi-static evolution of shape-memory materials. Math. Models Meth. Appl. Sci. 18 (2008) 125-164.

[10] F. Auricchio, A. Reali and U. Stefanelli, A macroscopic 1D model for shape memory alloys including asymmetric behaviors and transformation-dependent elastic properties. Comput. Methods Appl. Mech. Eng. 198 (2009) 1631-1637.

[11] A.-L. Bessoud and U. Stefanelli, A three-dimensional model for magnetic shape memory alloys. Preprint IMATI-CNR 27PV09/20/0 (2009).

[12] M. Brokate and J. Sprekels, Hysteresis and phase transitions, Applied Mathematical Sciences 121. Springer-Verlag, New York (1996).

[13] P. Colli, Global existence for the three-dimensional Frémond model of shape memory alloys. Nonlinear Anal. 24 (1995) 15651579.

[14] P. Colli and J. Sprekels, Global existence for a three-dimensional model for the thermodynamical evolution of shape memory alloys. Nonlinear Anal. 18 (1992) 873-888.

[15] T.W. Duerig, A.R. Pelton, Eds., SMST-2003 Proceedings of the International Conference on Shape Memory and Superelastic Technology Conference. ASM International (2003).

[16] T.W. Duerig, K.N. Melton, D. Stökel and C.M. Wayman, Eds., Engineering aspects of shape memory alloys. ButterworthHeinemann (1990). 
[17] F. Falk, Martensitic domain boundaries in shape-memory alloys as solitary waves. J. Phys. C4 Suppl. 12 (1982) 3-15.

[18] F. Falk and P. Konopka, Three-dimensional Landau theory describing the martensitic phase transformation of shape-memory alloys. J. Phys. Condens. Matter 2 (1990) 61-77.

[19] M. Frémond, Matériaux à mémoire de forme. C. R. Acad. Sci. Paris Sér. II Méc. Phys. Chim. Sci. Univers Sci. Terre 304 (1987) 239-244.

[20] M. Frémond, Non-smooth Thermomechanics. Springer-Verlag, Berlin (2002).

[21] S. Govindjee and C. Miehe, A multi-variant martensitic phase transformation model: formulation and numerical implementation. Comput. Methods Appl. Mech. Eng. 191 (2001) 215-238.

[22] D. Helm and P. Haupt, Shape memory behaviour: modelling within continuum thermomechanics. Int. J. Solids Struct. 40 (2003) 827-849.

[23] M. Hilpert, On uniqueness for evolution problems with hysteresis, in Mathematical Models for Phase Change Problems, J.F. Rodrigues Ed., Birkhäuser, Basel (1989) 377-388.

[24] K.H. Hoffmann, M. Niezgódka and S. Zheng, Existence and uniqueness to an extended model of the dynamical developments in shape memory alloys. Nonlinear Anal. 15 (1990) 977-990.

[25] P. Krejčí, Hysteresis, Convexity and Dissipation in Hyperbolic Equations, GAKUTO Int. Series Math. Sci. Appl. 8. Gakkotosho, Tokyo (1996).

[26] P. Krejčí and U. Stefanelli, Existence and nonexistence for the full thermomechanical Souza-Auricchio model of shape memory wires. Preprint, IMATI-CNR, 12PV09/10/0 (2009).

[27] D.C. Lagoudas, P.B. Entchev, P. Popov, E. Patoor, L.C. Brinson and X. Gao, Shape memory alloys, Part II: Modeling of polycrystals. Mech. Materials 38 (2006) 391-429.

[28] V.I. Levitas, Thermomechanical theory of martensitic phase transformations in inelastic materials. Int. J. Solids Struct. 35 (1998) 889-940.

[29] G.A. Maugin, The thermomechanics of plasticity and fracture, Cambridge Texts in Applied Mathematics. Cambridge University Press, Cambridge (1992).

[30] A. Mielke and A. Petrov, Thermally driven phase transformation in shape-memory alloys. Adv. Math. Sci. Appl. 17 (2007) 160-182.

[31] A. Mielke, L. Paoli and A. Petrov, On existence and approximation for a 3D model of thermally-induced phase transformations in shape-memory alloys. SIAM J. Math. Anal. 41 (2009) 1388-1414.

[32] A. Mielke, L. Paoli, A. Petrov and U. Stefanelli, Error estimates for discretizations of a rate-independent variational inequality. WIAS Preprint n. 1407 (2009)

[33] A. Mielke, L. Paoli, A. Petrov and U. Stefanelli, Error control for space-time discretizations of a 3D model for shape-memory materials, in Proceedings of the IUTAM Symposium on Variational Concepts with Applications to the Mechanics of Materials (Bochum 2008), IUTAM Bookseries, Springer (2009).

[34] I. Pawłow, Three-dimensional model of thermomechanical evolution of shape memory materials. Control Cybernet. 29 (2000) $341-365$

[35] B. Peultier, T. Ben Zineb and E. Patoor, Macroscopic constitutive law for SMA: Application to structure analysis by FEM. Materials Sci. Eng. A 438-440 (2006) 454-458.

[36] P. Popov and D.C. Lagoudas, A 3-D constitutive model for shape memory alloys incorporating pseudoelasticity and detwinning of self-accommodated martensite. Int. J. Plast. 23 (2007) 1679-1720.

[37] B. Raniecki and Ch. Lexcellent, $R_{L}$ models of pseudoelasticity and their specification for some shape-memory solids. Eur. J. Mech. A Solids 13 (1994) 21-50.

[38] S. Reese and D. Christ, Finite deformation pseudo-elasticity of shape memory alloys - Constitutive modelling and finite element implementation. Int. J. Plast. 28 (2008) 455-482.

[39] T. Roubíček, Models of microstructure evolution in shape memory alloys, in Nonlinear Homogenization and its Appl. to Composites, Polycrystals and Smart Materials, P. Ponte Castaneda, J.J. Telega, B. Gambin Eds., NATO Sci. Series II/170, Kluwer, Dordrecht (2004) 269-304.

[40] A.C. Souza, E.N. Mamiya and N. Zouain, Three-dimensional model for solids undergoing stress-induced phase transformations. Eur. J. Mech. A Solids 17 (1998) 789-806.

[41] U. Stefanelli, Analysis of a variable time-step discretization for the Penrose-Fife phase relaxation problem. Nonlinear Anal. 45 (2001) 213-240.

[42] P. Thamburaja and L. Anand, Polycrystalline shape-memory materials: effect of crystallographic texture. J. Mech. Phys. Solids 49 (2001) 709-737.

[43] F. Thiebaud, Ch. Lexcellent, M. Collet and E. Foltete, Implementation of a model taking into account the asymmetry between tension and compression, the temperature effects in a finite element code for shape memory alloys structures calculations. Comput. Materials Sci. 41 (2007) 208-221.

[44] A. Visintin, Differential Models of Hysteresis, Applied Mathematical Sciences 111. Springer, Berlin (1994).

[45] S. Yoshikawa, I. Pawłow and W.M. Zajączkowski, Quasi-linear thermoelasticity system arising in shape memory materials. SIAM J. Math. Anal. 38 (2007) 1733-1759. 